\title{
Optimal vitamin D supplementation strategies
}

\author{
Roger Bouillon ${ }^{1}$
}

Received: 26 December 2016 / Accepted: 19 January 2017 / Published online: 1 February 2017

(C) Springer Science+Business Media New York 2017

Humans survived evolution without vitamin D-rich food or without vitamin D supplementation until vitamin D deficiency, rickets, became a major health problem from the 17 th century onwards [1]. During the $20^{\text {th }}$ century, it became clear that exposure to sunlight and UVB should be avoided (infants and young children), or limited because of life time risks of skin damage or skin cancers. Moreover, voluntary avoidance (e.g. for cultural or religious reasons) of sun exposure or mismatch between skin color and climate (dark skinned immigrants living in Northern or Southern latitudes) severely limits the endogenous production of vitamin $\mathrm{D}$ well below the normal vitamin $\mathrm{D}$ requirements. This cannot be compensated by promoting natural vitamin D-rich food as there is simply not enough fatty fish in the ocean. Therefore vitamin D supplementation (either as vitamin D enriched food or by intake of supplements) will remain necessary for all infants and young children, for most elderly subjects and for other risk groups $[2,3]$. The major remaining questions are the overall health implications of vitamin D beyond bone health, the precise requirements (and thus the definition of vitamin $\mathrm{D}$ deficiency and the associated serum $250 H D$ concentrations) and the practical modalities to implement and maintain this supplementation for many years or for a lifetime to millions or even billions of people in a cost efficient way. Vitamin D is rapidly cleared from the circulation after its oral intake but it has nevertheless a long biological life time and its major metabolite, 25OHD, has a half-life of about

Roger Bouillon

roger.bouillon@kuleuven.be

1 Department of Endocrinology, KU Leuven, Gasthuisberg, Leuven 3000, Belgium
2 weeks [4]. As to enhance compliance, high dose intermittent therapy (once every 6-12 months) has been introduced in the past but several recent studies documented either transient hypercalcemia in children [5] or transient increased risks of falls or fractures [6, 7] and therefore this strategy is no longer a valid option. Daily, weekly or monthly use of vitamin D (in equivalent cumulative dosage) could enhance long term compliance depending on the behavior or preference of the target groups. Whether such intermittent therapy generates the same vitamin D status has been studied by a group of Hungarian scientists [8]. They randomized modestly vitamin $\mathrm{D}$ deficient adults (mean age 53 years; mean baseline $25 \mathrm{OHD}$ of $\sim 13 \mathrm{ng} / \mathrm{ml}$ ) to receive either daily (1000 IU), weekly (7000 IU) or monthly (30,000 IU) doses of vitamin $\mathrm{D}_{3}$ and found very similar increase in the serum 25OHD after 3 months. This conclusion was essentially the same whether or not the data were corrected for several potentially confounding factors. The increase in serum 25OHD was about $1.3 \mathrm{ng} / 100 \mathrm{IU}$, in line with most previous studies in subjects with a similarly low baseline vitamin D status. The results confirm a previous (equally small) study in elderly subjects (mean age of 80 years [9]), whereas others found a slightly lower efficacy of monthly compared to daily or weekly doses [10]. Collectively, however, these reports confirm that these strategies yield essentially similar results especially when taking into account the wide interindividual differences. Such choice of options thus may guide clinicians to find optimal long-term compliance. Although Hollis claimed that steady supply of vitamin D may be more beneficial than intermittent dosage [11], such intermittent therapy may resemble the natural intermittent endogenous production of vitamin depending on the intermittent exposure to natural sunlight or access to vitamin $\mathrm{D}$ rich food sources. The dosage 
selected by Takacs et al. [8] is however rather unfortunate as it does not correspond to the daily dosages recommended by several international guidelines (Institute Of Medicine or IOM, Nordic and DACH (German speaking countries) countries, Australia/New Zealand, as reviewed in [12]). In addition, they used a 25OHD method that is neither NIST nor DEQAS-validated, and therefore they need to remeasure at least $20 \%$ of the serum samples using a wellvalidated method [13] before these data can become internationally useful. Intermittent use of vitamin $\mathrm{D}_{2}$ (ergocalciferol) may not be equally efficient as it has a shorter half-life than vitamin $D_{3}$, and its general biological equivalence to vitamin $\mathrm{D}_{3}$ is under revision $[14,15]$. The results of Takacs et al. are thus useful with regard to the efficacy of daily, weekly or monthly dosage of vitamin $\mathrm{D}_{3}$ as to generate similar serum 250 HD concentrations. Whether that also applies to similar beneficial health outcomes needs to be studied in larger scale studies. This study is also a reminder for all investigators that quality control of measurements of the serum 250HD are now absolutely essential as to allow international comparison of biochemical or clinical benefits. In addition, future studies should be designed as to try to explain the genetic, metabolic or environmental mechanisms responsible for the wide variability of vitamin $\mathrm{D}$ status at baseline or after vitamin $\mathrm{D}$ supplementation

\section{Compliance with ethical standards}

Conflict of interest The authors declare that they have no competing interest.

\section{References}

1. R. Bouillon, Vitamin D: from photosynthesis, metabolism, and action to clinical applications, in Endocrinology: adult and pediatric, ed. by J.L. Jameson, L.J. De Groot (Saunders/Elsevier, Philadelphia, 2016), pp. 1018-1038

2. R. Bouillon, N.M. Van Schoor, E. Gielen, S. Boonen, C. Mathieu, D. Vanderschueren, P. Lips, Optimal vitamin D status: a critical analysis on the basis of evidence-based medicine. J. Clin. Endocrinol. Metab. 98(8), E1283-E1304 (2013)
3. H. Fuleihan Gel, R. Bouillon, B. Clarke, M. Chakhtoura, C. Cooper, M. McClung, R.J. Singh, Serum 25-hydroxyvitamin D Levels: variability, knowledge gaps, and the concept of a desirable range. J. Bone Miner. Res. 30(7), 1119-1133 (2015)

4. K.S. Jones, S. Assar, D. Harnpanich, R. Bouillon, D. Lambrechts, A. Prentice, I. Schoenmakers, $25(\mathrm{OH}) \mathrm{D} 2$ half-life is shorter than $25(\mathrm{OH}) \mathrm{D} 3$ half-life and is influenced by DBP concentration and genotype. J. Clin. Endocrinol. Metab. 99(9), 3373-3381 (2014)

5. H. Mittal, S. Rai, D. Shah, S.V. Madhu, G. Mehrotra, R.K. Malhotra, P. Gupta, 300,000 IU or 600,000 IU of oral vitamin D3 for treatment of nutritional rickets: a randomized controlled trial. Indian Pediatr. 51(4), 265-272 (2014)

6. K.M. Sanders, A.L. Stuart, E.J. Williamson, J.A. Simpson, M.A. Kotowicz, D. Young, G.C. Nicholson, Annual high-dose oral vitamin $\mathrm{D}$ and falls and fractures in older women: a randomized controlled trial. JAMA 303(18), 1815-1822 (2010)

7. H. Smith, F. Anderson, H. Raphael, P. Maslin, S. Crozier, C. Cooper, Effect of annual intramuscular vitamin D on fracture risk in elderly men and women--a population-based, randomized, double-blind, placebo-controlled trial. Rheumatology 46(12), 1852-1857 (2007)

8. I. Takacs, B.E. Toth, L. Szekeres, B. Szabo, B. Bakos, P. Lakatos, Randomized clinical trial to comparing efficacy of daily, weekly and monthly administration of vitamin D3. Endocrine. (2016). doi:10.1007/s12020-016-1137-9

9. S. Ish-Shalom, E. Segal, T. Salganik, B. Raz, I.L. Bromberg, R. Vieth, Comparison of daily, weekly, and monthly vitamin D3 in ethanol dosing protocols for two months in elderly hip fracture patients. J. Clin. Endocrinol. Metab. 93(9), 3430-3435 (2008)

10. V. Chel, H.A. Wijnhoven, J.H. Smit, M. Ooms, P. Lips, Efficacy of different doses and time intervals of oral vitamin D supplementation with or without calcium in elderly nursing home residents. Osteoporos. Int. 19(5), 663-671 (2008)

11. B.W. Hollis, C.L. Wagner, Clinical review: The role of the parent compound vitamin $\mathrm{D}$ with respect to metabolism and function: Why clinical dose intervals can affect clinical outcomes. J. Clin. Endocrinol. Metab. 98(12), 4619-4628 (2013)

12. R. Bouillon, Comparative analysis of Nutritional guidelines for vitamin D. Nat. Rev. Endocrinol. (2017) in press

13. C.T. Sempos, R.A. Durazo-Arvizu, N. Binkley, J. Jones, J.M. Merkel, G.D. Carter, Developing vitamin D dietary guidelines and the lack of 25-hydroxyvitamin D assay standardization: the everpresent past. J. Steroid Biochem. Mol. Biol. 164, 115-119 (2016)

14. A. Shieh, R.F. Chun, C. Ma, S. Witzel, B. Meyer, B. Rafison, L. Swinkels, T. Huijs, S. Pepkowitz, B. Holmquist, M. Hewison, J.S. Adams, Effects of high-dose vitamin D2 versus D3 on total and free 25-hydroxyvitamin D and markers of calcium balance. J. Clin. Endocrinol. Metab. 101(8), 3070-3078 (2016)

15. R. Bouillon, L. Verlinden, A. Verstuyf, Is vitamin D2 really bioequivalent to vitamin D3? Endocrinology 157(9), 3384-3387 (2016) 